\title{
Neuroprotective Effects of Purslane Seeds against Adverse Effects Induced by Experimental Hyperlipidemia on Frontal Cortex and Cerebellum in Young Male Albino Rats
}

\author{
El Sayed Aly Mohamed Metwally ${ }^{1, ~}$, Fardous Soror Karawya ${ }^{2}$ \\ ${ }^{1}$ Department of Anatomy and Embryology, Faculty of Medicine, Alexandria University, Alexandria, Egypt \\ ${ }^{2}$ Department of Histology and Cell biology, Faculty of Medicine, Alexandria University, Alexandria, Egypt \\ Email address: \\ sayedmetwally2020@yahoo.com (E. S. A. M. Metwally)
}

\section{To cite this article:}

El Sayed Aly Mohamed Metwally, Fardous Soror Karawya. Neuroprotective Effects of Purslane Seeds against Adverse Effects Induced by Experimental Hyperlipidemia on Frontal Cortex and Cerebellum in Young Male Albino Rats. International Journal of Clinical and Experimental Medical Sciences. Vol. 1, No. 3, 2015, pp. 46-59. doi: 10.11648/j.ijcems.20150103.14

\begin{abstract}
Background. Hyperlipidemia is characterized by abnormally elevated levels of lipids and is positively associated with cerebrovascular diseases. Alzheimer's disease (AD) is a chronic brain disorder characterized by cognitive impairment, inflammation, $\beta$-amyloid deposition, and vascular damage. Recent studies have shown that high cholesterol levels are linked to the pathology of AD. Purslane seeds are medicinal plants rich in unsaturated fatty acids (omega-3), antioxidants, and fibers. They are known to have antidiabetic and antiatherogenic activities. The aim of this research is to study the structural alterations occurring in the frontal cortex and cerebellum after feeding high cholesterol diet, and the possible protective role of purslane seeds. Materials and methods. Forty male albino rats were used in the study. They were divided into three groups; control group (20 rats), hypercholesterolemic group (10 rats) who were fed the balanced diet supplemented with cholesterol at a dose of 2 $\mathrm{gm} / 100 \mathrm{gm}$ diet, and protected group (10 rats) who were fed the same previous hypercholesterolemic diet concomitant with purslane seed 20\% (20 gm/100 ml water). After 3 months, blood samples were collected from all rats for biochemical estimation and fresh specimens were taken from the frontal cortex and cerebellum of each rat and processed for; light microscopic examination using H\&E and Orcein stains. Results. Significant increase of serum triglyceride (TG), total cholesterol (TC), and low-density lipoprotein (LDL-C) levels in hyperlipidemic rats were detected. Associated structural changes in the frontal cortex of the rats were evident as pyknotic nuclei of degenerated neurons, loss of neuron, dilation and congestion of blood vessels, expanded perivascular space, and increased vacuolar spaces of neuropil. The cerebellar cortex of the rats fed on high cholesterol diet revealed pyknotic nuclei of degenerated neuron and decrease in the number of Purkinje and granule cells compared to the control group. Concomitant administration of purslane seeds revealed evident amelioration of biochemical and most of the structural changes. Conclusion. We concluded that a high cholesterol diet has deleterious biochemical changes associated with structural alteration in the frontal cortex and cerebellum and purslane seeds ameliorate most of these changes.
\end{abstract}

Keywords: High Cholesterol Diet, Neurotoxicity, Oxidative Stress, Purslane Seeds, Male Rats

\section{Introduction}

According to the estimates of the World Health Organization (WHO), overweight and obesity are the fifth risk factor for mortality worldwide, causing approximately 2.8 million deaths per year. Obesity is fast, becoming a medical condition of global concern, particularly due to its consistent association with increased prevalence of cardiovascular diseases, diabetes mellitus, hypertension and some forms of cancers. ${ }^{1-3}$ Hypercholesterolemia (HChol) is considered as one of the most familiar metabolic disorders and is closely associated with obesity. Studies demonstrated that HChol is significantly associated with oxidative stress and the generation of reactive oxygen species (ROS) ${ }^{4-6}$ It is well established that HChol induces inflammation in the brain. ${ }^{7,8}$ Chronic treatment of rats with cholesterol markedly increased microglial immunoreactivity and levels of several inflammatory markers in the cortex, reduced cholinergic neurons and impaired cognition. ${ }^{9}$ Furthermore, hypercholesterolemia in rats displays blood brain barrier (BBB) disruptions in the cortex visualized by an increased anti-rat IgG immunoreactivity. ${ }^{10}$ Also, hypercholesterolemia has been described as a risk factor for vascular dementia 
(VaD) and Alzheimer's disease (AD). ${ }^{11,12}$ Therefore, it is necessary to search for effective approaches to control hypercholesterolemia and the associated brain complications.

Weight reduction, via dietary modulation is therefore the target of choice of many therapeutic measures. Conventional drugs promote about $5-10 \%$ loss of weight, and best, only when used in conjunction with diet, exercise, and behaviour change regimens. ${ }^{13}$ The reported minor and grave side effects of the drugs, as well as weight rebounds when discontinued, have necessitated a new dimensional approach into the search for anti-obesity and antihypercholesterolemic agents. $^{14}$

Medicinal plants are widely used in the management of diseases all over the world. Herbal medicines have reported significant improvement in controlling body weight, without any noticeable adverse effects. ${ }^{13}$ Portulaca oleracea (Portulacaceae family), (also referred to the common; Purslane), is listed in the WHO as one of the most used medicinal plants and has been given the term global panacea. ${ }^{14-16}$ It is widespread as a weed and has been ranked the eighth most common plant in the world. ${ }^{17}$ Portulaca oleracea, or purslane, is a good source of biologically active compounds, including omega-3 fatty acids, $\beta$-carotene, amino acids, $\alpha$-tocopherols, ascorbic acid, glutathione, alkaloids, antioxidant vitamins (such as vitamin A, vitamin C, and some vitamin B and carotenoids), as well as dietary minerals, such as magnesium, calcium, potassium, iron, and flavonoid compounds. ${ }^{18-21}$

Based on these issues and concerns, the aim of the present study was to investigate the structural alterations in the frontal cortex and cerebellum due to a high cholesterol diet, and to see whether purslane seeds can ameliorate this possible damage.

\section{Material \& Methods}

\subsection{Material and Subjects}

This study was carried out on 40 adult male albino rats weighing about 100-150 grams (gm). Their average ages were from 3-4 months. Animals were maintained under standard housing conditions, and were allowed free access to standard laboratory food and water. Guidelines for care and use of animals approved by the Animal House Center, Faculty of Medicine, University of Alexandria, were followed. The animals were randomly divided into three groups as follows:

1. Group I: control group, which was further subdivided into two equal subgroups 10 animals each.

a. Subgroup Ia, received standard diet.

b. Subgroup Ib received purslane seeds in water orally by orogastric tube $2 \mathrm{ml} /$ day for three months.

2. Group II: experimental group, which included 10 rats who received high fat, high cholesterol (HFHC) diet. It consisted of a high fatty diet (standard diet together with coconut oil, palm oil and egg yolk) and cholesterol
$2 \%$ for three months to induce hyperlipidemia. ${ }^{22}$

3. Group III: protected group, which consisted of 10 rats who received simultaneously both $\mathrm{HFHC}$ diet and purslane seeds as in subgroup Ib for three months.

Cholesterol used in the experiment was purchased from the Oxford Chemical Company in the form of powder. It was dissolved in coconut oil.

Purslane seeds were purchased from the 'local market.' It was crushed by an electric grinder and stored at $4^{\circ} \mathrm{C}$ prior to its use and then was dissolved in water ( $20 \mathrm{gm} / 100 \mathrm{ml}$ water).

At the end of the experimental period (3 months), rats were sacrificed after overnight fasting and the blood of each rat was taken from the abdominal aorta under ether anesthesia. The serum was separated by allowing the blood sample to be left for 15 minutes at a temperature of $25^{\circ} \mathrm{C}$, then centrifuged at $3000 \mathrm{RPM}$ for 20 minutes, then kept in plastic vials at $-20^{\circ} \mathrm{C}$. Serum was analyzed for the following biochemical parameters: triglyceride (TG), total cholesterol (TC), high-density lipoprotein-cholesterol (HDL-C) and low density lipoprotein-cholesterol (LDL-C) and determined by equations developed by Friedewald. ${ }^{23}$

Fresh specimens were taken from the frontal cortex and cerebellum of each rat. Each specimen was fixed in $10 \%$ formal saline and processed to get paraffin sections for light microscopic examination using:

1. Haemotoxylin and Eosin (H\&E) stain. ${ }^{24,25}$

2. Orcein stain. ${ }^{24,25}$

\subsection{Statistical Analysis}

The results obtained were analyzed using SPSS program (version 17.0) and expressed as mean and standard deviation (SD). Statistical significance $(p<0.05)$ among the groups was determined by one-way ANOVA.

\section{Results}

\subsection{General Appearance and Survival}

Observation of rats during the experimental period revealed that HFHC diet was well tolerated by the rats and no mortality was detected. Rats of all groups exhibited normal activities and had smooth and glossy fur.

\subsection{Lipid Profiles Level}

Comparison between lipid profiles in different studied groups has been shown in table1. Regarding triglyceride, it was found that there was a significant increase in the high cholesterol group than both control and high cholesterol with purslane seeds, while there was no significant difference between control and high cholesterol with purslane seeds. Total cholesterol showed a significant difference between the three groups, the highest value was found in cholesterol group, followed by high cholesterol with purslane seeds and the lowest value was in control group. HDL-C show a significant increase in control group than the other two groups, while there was no significant difference between high cholesterol group and high cholesterol with purslane 
48 El Sayed Aly Mohamed Metwally and Fardous Soror Karawya: Neuroprotective Effects of Purslane Seeds against Adverse Effects Induced by Experimental Hyperlipidemia on Frontal Cortex and Cerebellum in Young Male Albino Rats

seeds. LDL-C showed a significant difference between the three groups, the highest level was in high cholesterol group followed by high cholesterol with purslane seeds then the control group.

Table 1. Comparison between lipid profiles in different studied groups.

\begin{tabular}{lllll}
\hline Groups & TG & TC & HDL-C & LDL-C \\
\hline Control & $1.6 \pm$ & $2.9 \pm 0.51$ & $1.7 \pm 0.4$ & $0.88 \pm$ \\
& 0.26 & & & 0.146 \\
High cholesterol & $2.96 \pm$ & $4.29 \pm$ & $1.21 \pm$ & $2.49 \pm$ \\
High & 0.275 & 0.136 & 0.136 & 0.224 \\
cholesterol+purslane & $1.71 \pm$ & $3.24 \pm$ & $1.48 \pm$ & $1.42 \pm$ \\
seeds & 0.146 & 0.132 & 0.136 & 0.13 \\
ANOVA & 15.62 & 10.25 & 6.21 & 28.9 \\
P & $0.001^{*}$ & $0.001^{*}$ & $0.017^{*}$ & $0.001^{*}$ \\
P1 & $0.001^{*}$ & $0.001^{*}$ & $0.003^{*}$ & $0.001^{*}$ \\
P2 & 0.136 & $0.023^{*}$ & $0.041^{*}$ & $0.0084^{*}$ \\
P3 & $0.003^{*}$ & $0.002^{*}$ & 0.078 & $0.021^{*}$ \\
\hline
\end{tabular}

$\mathrm{P}$ is significant if $<0.05$

P1: Comparison between control group and high cholesterol group

P2: Comparison between control and high cholesterol + purslane seeds group P3: Comparison between high cholesterol group and + purslane seeds group TG: Triglyceride, TC: Total cholesterol, HDL-C: High density

lipoprotein-cholesterol, LDL-C: Low density lipoprotein-cholesterol

\subsection{Histological Results}

Haematoxylin and Eosin stain:

Group I (Control Group):

H\&E stained sections of the control rat showed the normal histological structure of both frontal cortex and cerebellum. The pia arachnoid was seen covering the brain surface. The frontal cortex appeared laminated with six different layers of variable thickness that are blended with each other. The amorphous

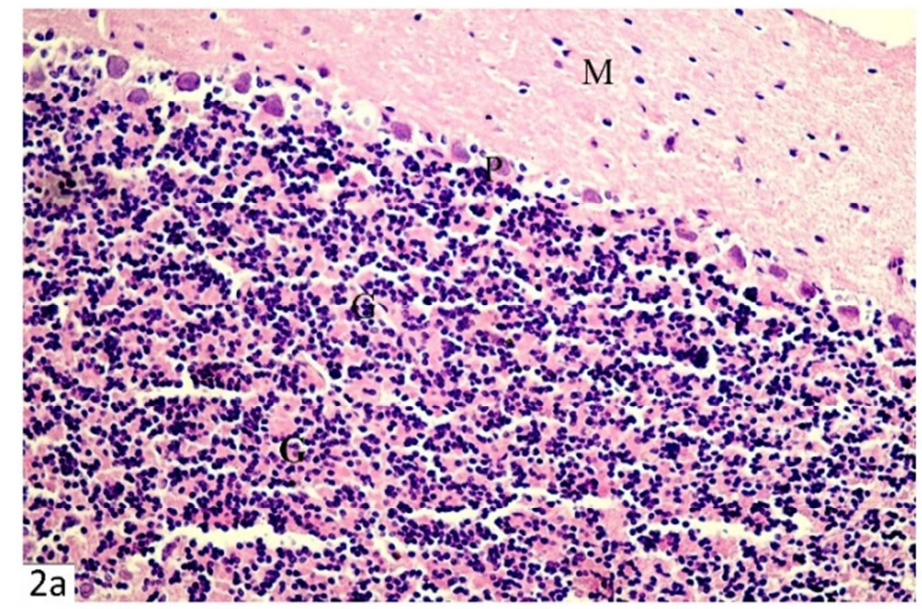

neuropil was revealed as a homogenous pale acidophilic background among the neuroglia and the neuronal cell bodies (Figure $1 \mathrm{a}, \mathrm{b}$ ).

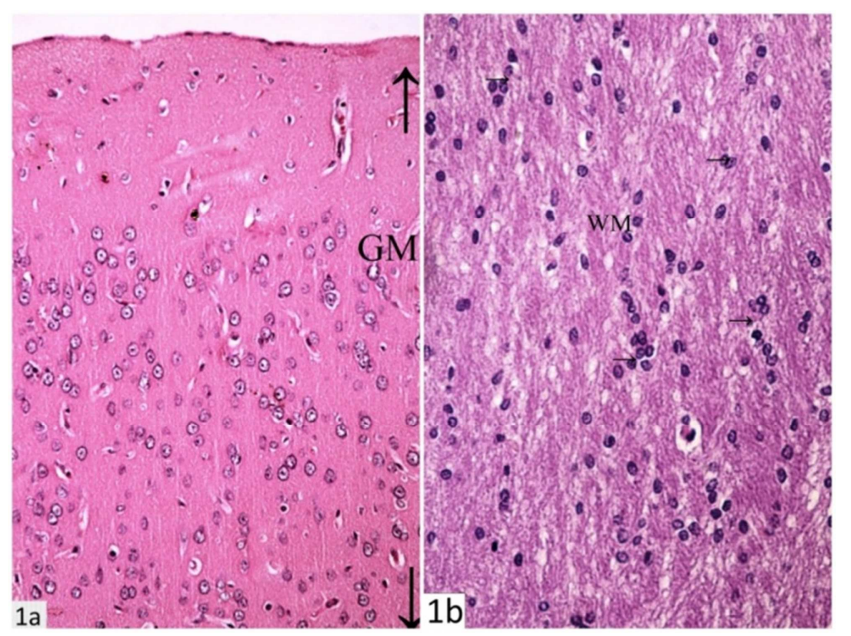

Figure 1. $\boldsymbol{a}, \boldsymbol{b}$. Photomicrographs of H\&E stained control rat frontal cortex showing: 1 a: Normally arranged layers of grey matter (GM) of frontal cortex and $1 \mathrm{~b}$ : demonstrating the white matter (WM) containing bundles of axons and nuclei of some neurons and neuroglial cells (个). H\&E stain X200.

The cerebellum of the control rat showed the foliated tree-like appearance with the grey matter externally located. The cerebellar cortex exhibited three well demarcated layers. The nuclei of the Bergmann astrocytes that ensheath the purkinje neurons were seen in between the purkinje cells. The cerebellar glomeruli composed of complex neuronal and glial processes are seen as scattered acidophilic areas among the granule cells (Figure $2 \mathrm{a}, \mathrm{b}$ ).

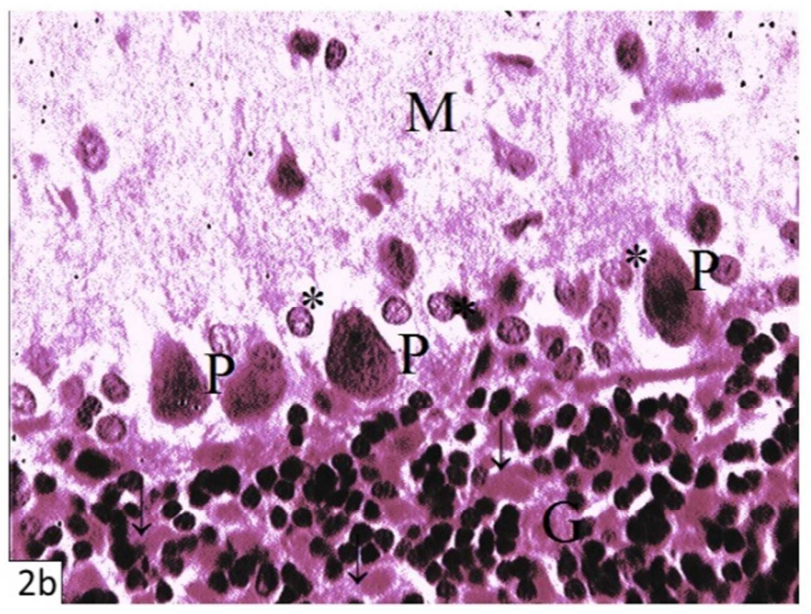

Figure 2. a, b. Photomicrographs of H\&E stained control rat cerebellar cortex showing: normally arranged cerebellar cortical layers, molecular layer (M) with few neurons, single row of pear-shaped purkinje cell layer $(P)$, the nuclei of the astrocytes of the purkinje layer (Bergmann cells) are revealed around the purkinje cell bodies (*) and inner granular layer with dense closely packed nuclei $(G)$. Note the cerebellar glomeruli occupying the spaces between the granule cells $(\uparrow)$. $H \&$ E stain $a$ X100 - b X400.

\section{Group II (Hypercholesterolemic Group):}

Frontal cortex of hypercholesterolemic rats showed various degrees of degenerative changes. This was proved by the many neurons and neuroglia, which appeared distorted with pyknotic nuclei and vacuolated cytoplasm compared to the control group. The congested dilated small blood vessels were also revealed within the loose neuropil. The neuropil appeared lightly stained and vacuolated (Figure $3 \mathrm{a}, \mathrm{b}, \mathrm{c} \&$ Figure $4 \mathrm{a}, \mathrm{b}$, $\mathrm{c}$, ), the grey matter of the frontal cortex revealed localized area of mononuclear cellular infiltration and numerous neuroglial 
cells (Figure $4 \mathrm{c}$ ), and the bundles of axons in the white matter were widely separated (Figure $4 \mathrm{~d}$ ).

The H\&E stained cerebellum of hypercholesterolemic rats exhibited loosening of molecular and granular layers, but the pattern of the folia was preserved. In addition, loss of Purkinje cells and granule cells were associated with the pyknotic nuclei of the degenerated cells, which were evident in the molecular and granular layers (Figure $5 \mathrm{a}, \mathrm{b}, \mathrm{c}$ ).
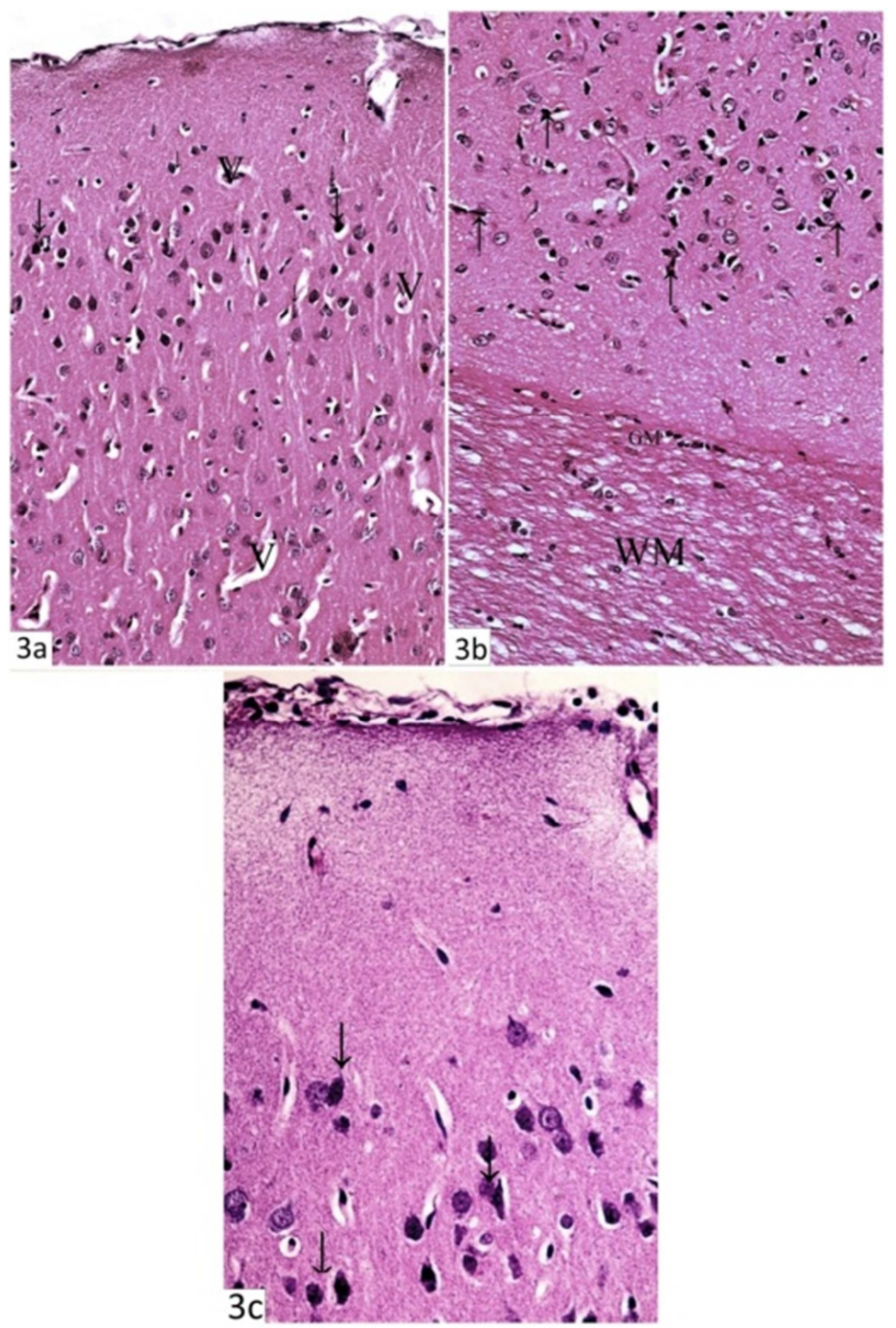

Figure $3 \boldsymbol{a}, \boldsymbol{b}, \boldsymbol{c}$. Photomicrographs of H\&E stained frontal cortex of hypercholesterolemic rats showing ill-defined cortical layers, distortion and decrease the number of neurons, numerous neuroglial cells $(\uparrow)$ and dilated blood vessels $(V)$. Note widely separated bundles of the axons in the white matter (WM). H\&E stain $a, b \times 200-c \times 400$. 
50 El Sayed Aly Mohamed Metwally and Fardous Soror Karawya: Neuroprotective Effects of Purslane Seeds against Adverse Effects Induced by Experimental Hyperlipidemia on Frontal Cortex and Cerebellum in Young Male Albino Rats

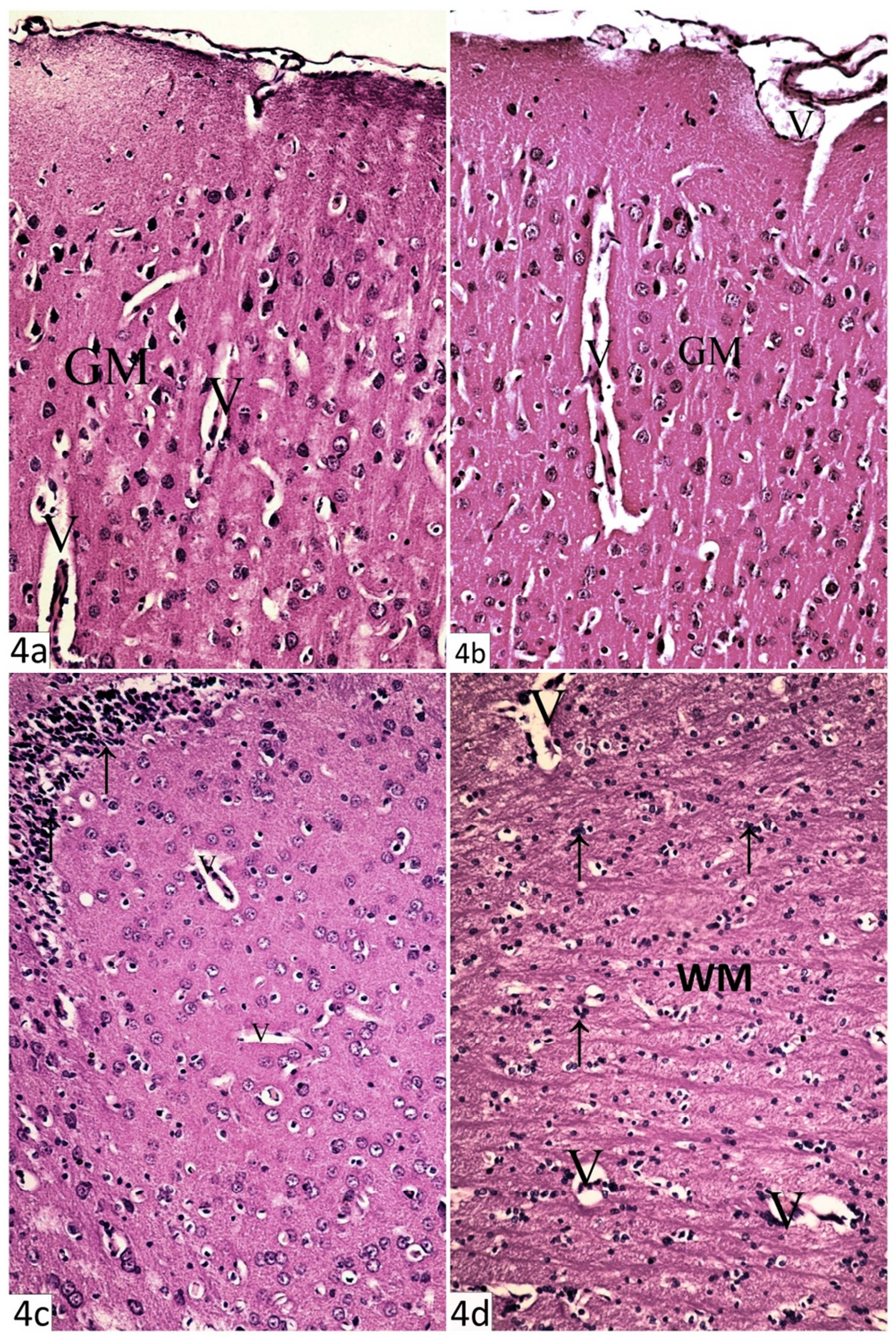

Figure $4 \boldsymbol{a}, \boldsymbol{b}, \boldsymbol{c}, \boldsymbol{d}$. Photomicrographs of H\&E stained frontal cortex of hypercholesterolemic rats showing: a,b: ill-defined layers of grey matter (GM) of frontal cortex, distortion of neurons, dilated blood vessels (V). 4 c: showing prominent neuroglial cells and mononuclear cellular infileration ( $\uparrow) .4 d$ : showing widely separated bundles of the axons in white matter (WM), numerous neuroglial cells with pyknotic nuclei $(\uparrow)$ and dilated blood vessels (V). H\&E stain X200. 

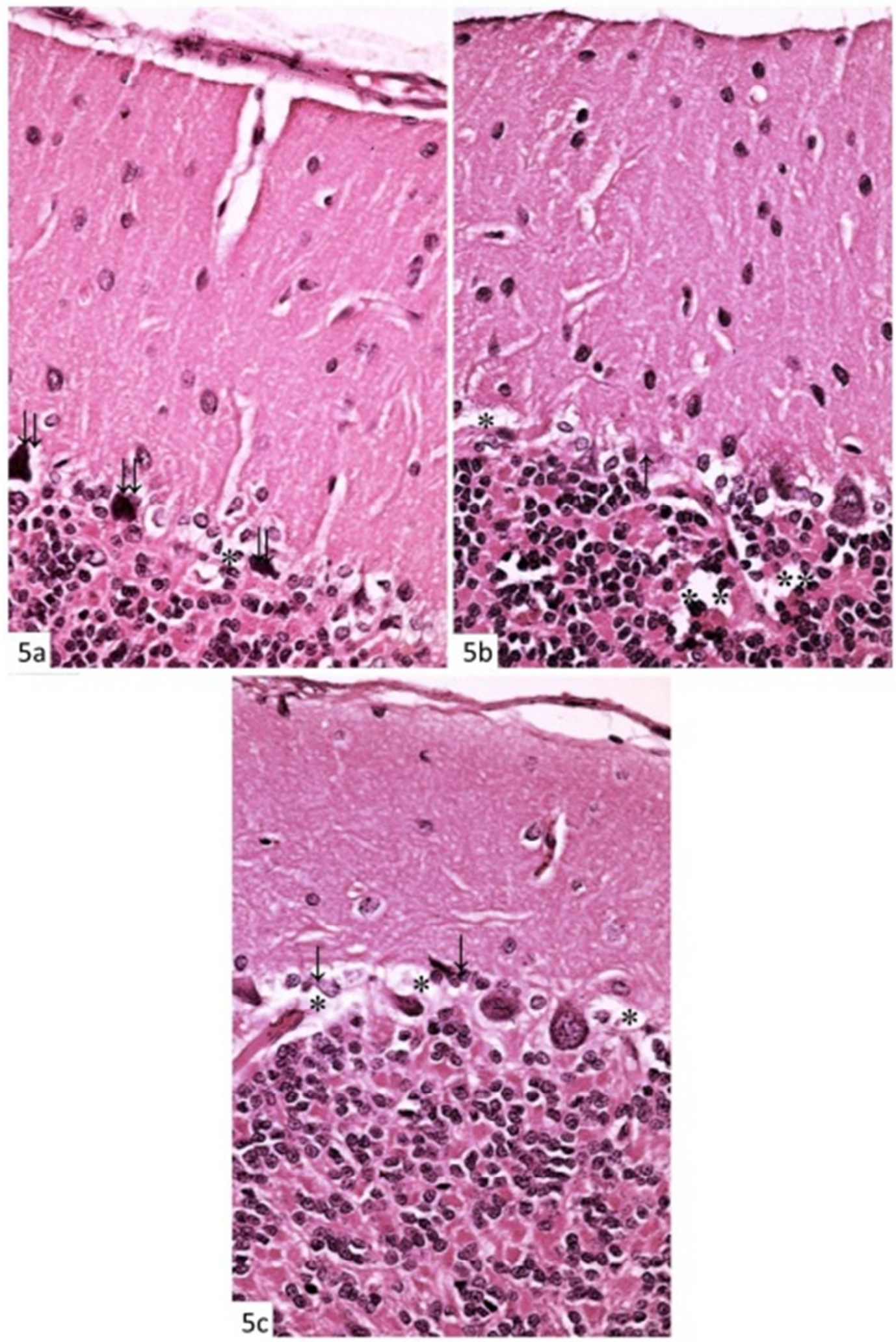

Figure $5 \boldsymbol{a}, \boldsymbol{b}, \boldsymbol{c}$. Photomicrographs of H\&E stained cerebellar cortex of hypercholesterolemic rats showing, distortion of purkinje cells, as well as cellular shrinkage $(\uparrow \uparrow)$ or complete destruction, vacuolation of the surrounding neuropil tissue $\left(^{*}\right)$, and some neuroglial cells accumulated around the purkinje cells $(\uparrow)$. Note some destruction and decrease in the number of granule cells $\left(^{* *}\right)$. H\&E stain X200.

\section{Group III (Protected Group):}

Frontal cortex of the protected group revealed more or less the control pattern with well-arranged layer except mild dilation of the cortical blood vessels and prominent nuclei of neuroglial cells (Figure $6 \mathrm{a}, \mathrm{b}$ ). The white matter of the frontal cortex revealed mild separation of the bundles of axons and many neuroglial cells (Figure $6 \mathrm{c}, \mathrm{d}$ ). 
52 El Sayed Aly Mohamed Metwally and Fardous Soror Karawya: Neuroprotective Effects of Purslane Seeds against Adverse Effects Induced by Experimental Hyperlipidemia on Frontal Cortex and Cerebellum in Young Male Albino Rats

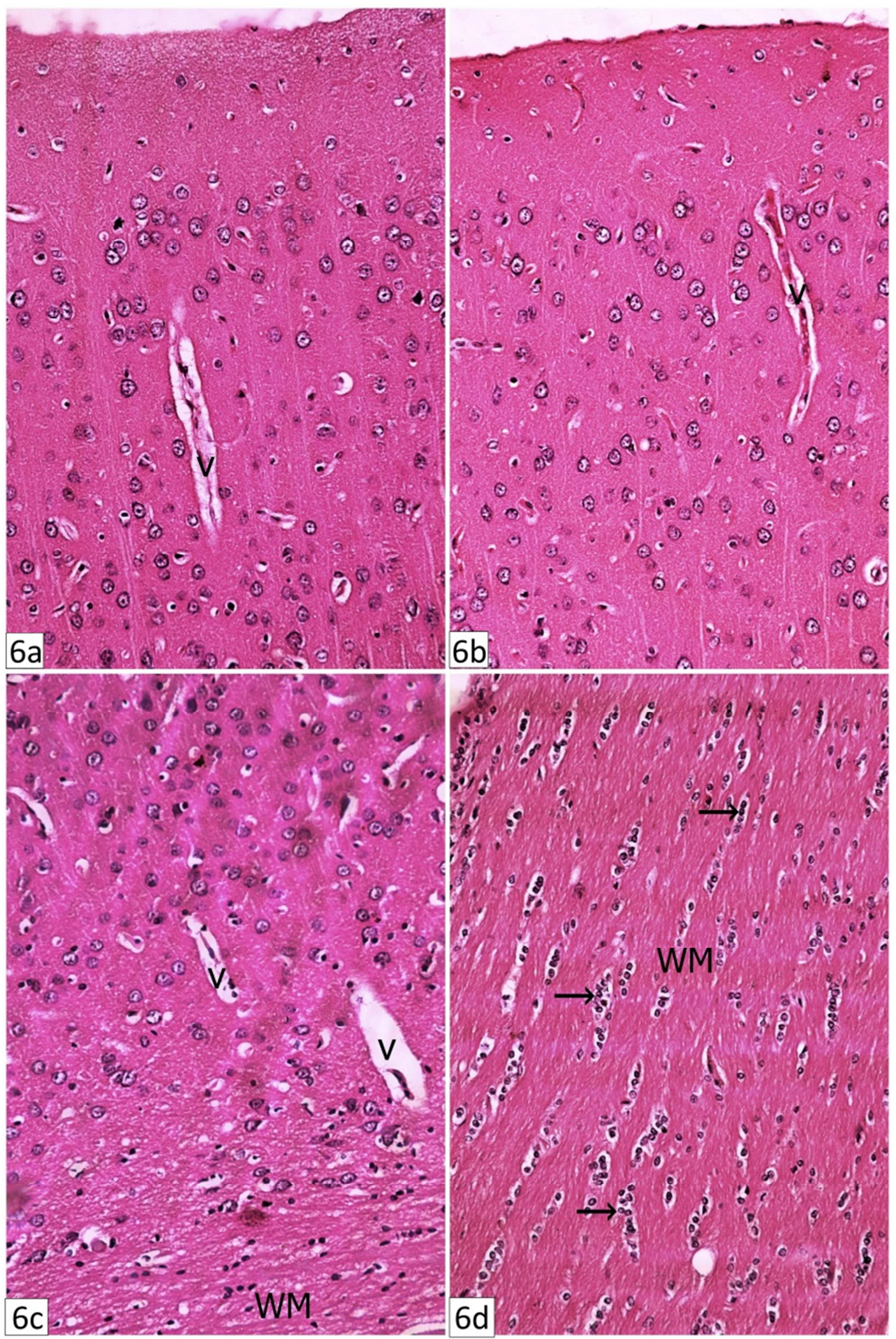

Figure $\mathbf{6} \boldsymbol{a}, \boldsymbol{b}, \boldsymbol{c}, \boldsymbol{d}$. Photomicrographs of $H \& E$ stained frontal cortex of the protected group (received purslane seeds concomitant with high cholesterol diet). a,b: showing more or less normal architecture of the frontal cortex except mild dilation of blood vessels $(V)$. $b, c$ : revealed numerous neuroglial ( $\uparrow)$ cells in white matter (WM). H\&E stain X200.

The H\&E stained cerebellum of the protected group revealed some destruction of purkinje cells and pericellular vacuolation (Figure $7 \mathrm{a}, \mathrm{b}$ ). 


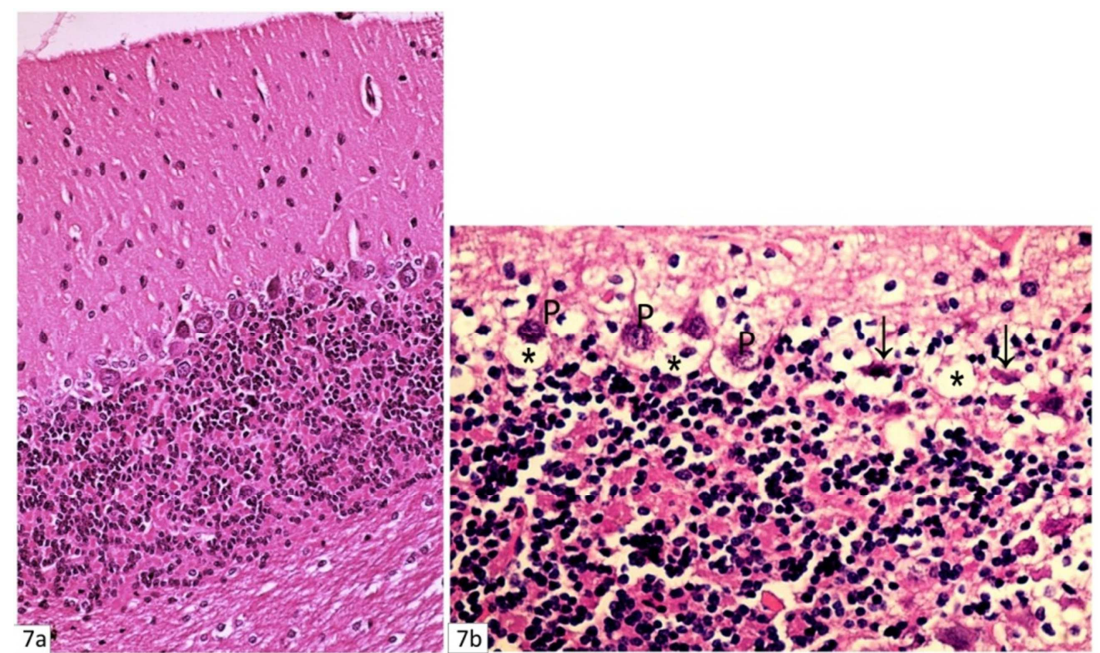

Figure $7 \boldsymbol{a}, \boldsymbol{b}$. Photomicrographs of H\&E stained cerebellar cortex of the protected group (received purslane seeds concomitant with high cholesterol diet) showing mild disorganization of the purkinje cell layer $(P)$. Some purkinje cells have normal shape while others are shrunken $(\uparrow)$. Note pericellular vacuolation (*). $H \& E$ stain a X200-bX400.
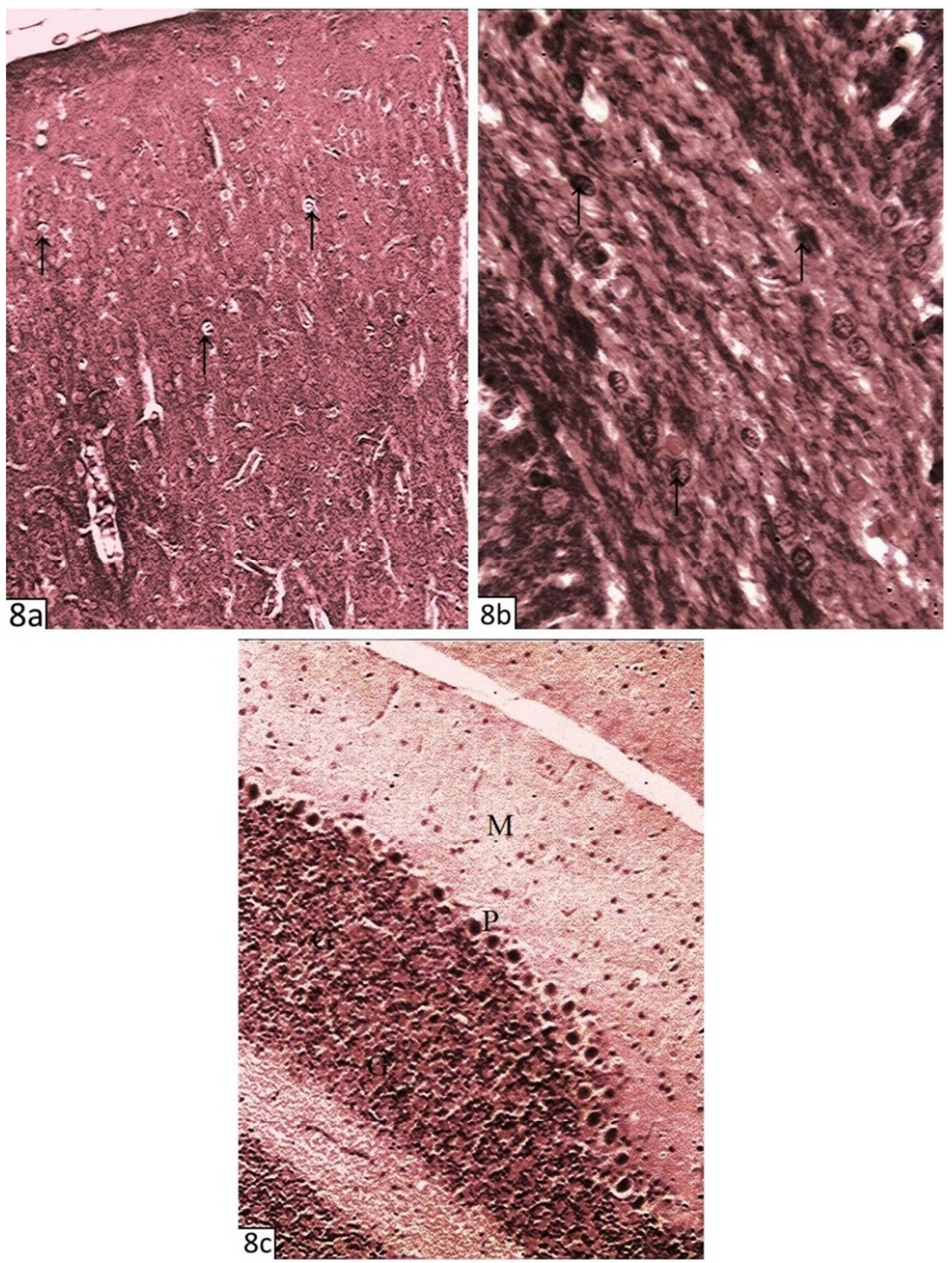

Figure 8 a,b,c. Photomicrographs of orcein stained control rat frontal and cerebellar cortex showing; 8 a: normal staining (red brown) neuropil tissue and negative staining nuclei of neurons and neuroglial $(\uparrow) .8 \mathrm{~b}$ : showing normal staining of bundles of axon associated with the nuclei of some neuroglial cells ( $\uparrow) .8$ c: showing the characteristic three layers of cerebellar cortex. Molecular (M), Purkinje (P), and granular (G) layers. Orcein stain X200. 
54 El Sayed Aly Mohamed Metwally and Fardous Soror Karawya: Neuroprotective Effects of Purslane Seeds against Adverse Effects Induced by Experimental Hyperlipidemia on Frontal Cortex and Cerebellum in Young Male Albino Rats

Orcein stain:

Orcein stained sections of the control rats frontal cortex revealed brownish red background. The neurons and neuroglia exhibited almost unstained nuclei (Figure 8 a). The white matter of the frontal cortex showing normal staining pattern of the axons associated with nuclei of neurons and neuroglial cells (Figure $8 \mathrm{~b}$ ). Orcein stained sections of the control rats cerebellar cortex showed a normal staining pattern of the three layers (Figure $8 \mathrm{c}$ ).

Orcein stained sections of the frontal cortex of hypercholesterolemic rats revealed pale staining and vacuolated background, darkly stained cell bodies of neurons and neuroglia or unstained nuclei, localized area of vacuolation associated with congested blood vessels (Figure 9 a, b, c, d).
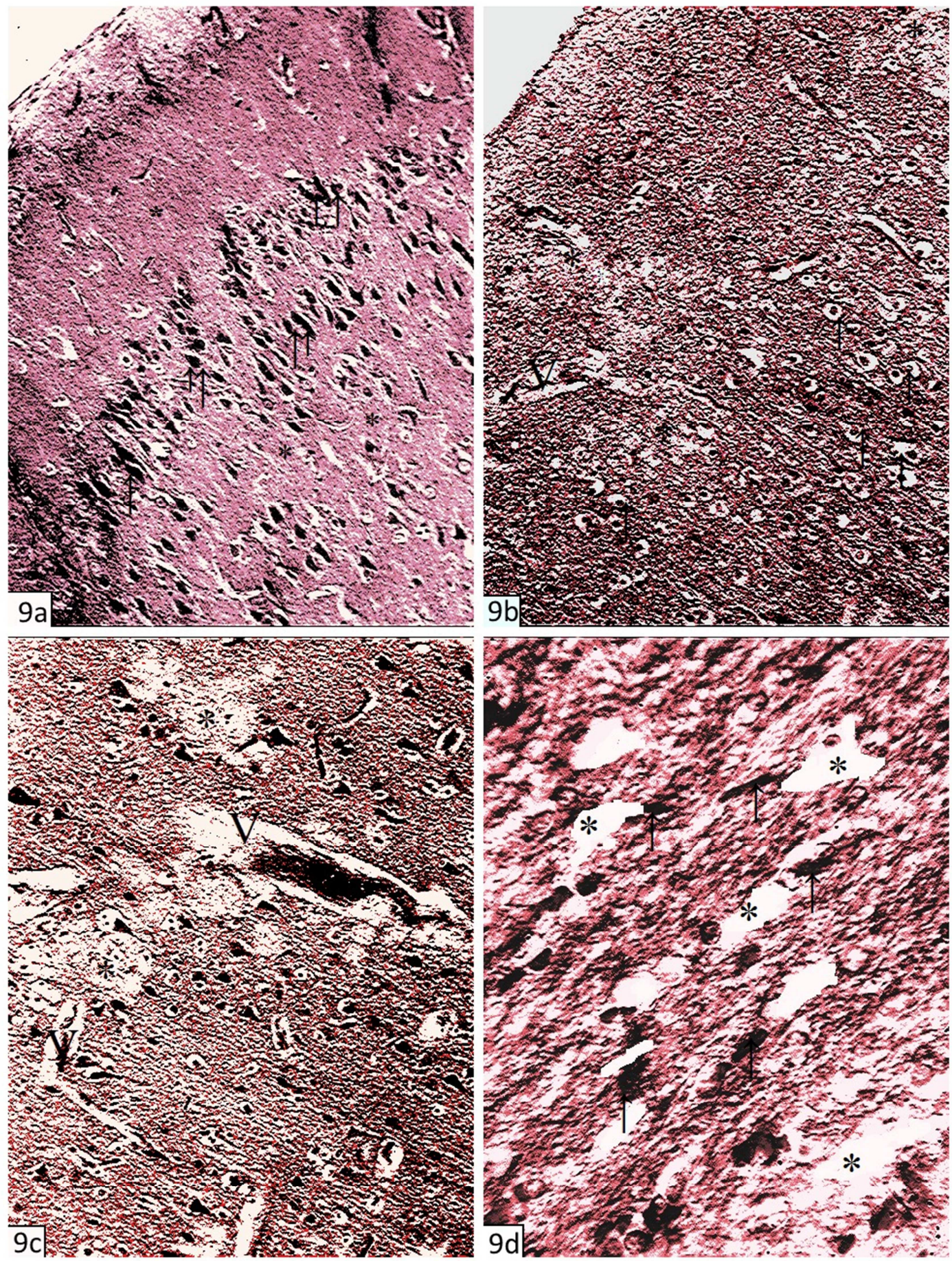

Figure $9 \boldsymbol{a}, \boldsymbol{b}, \boldsymbol{c}, \boldsymbol{d}$. Photomicrographs of orcein stained frontal cortex of hypercholesterolemic rats showing 9 a: dark staining ( $\uparrow \uparrow)$ and few in number of neurons. $9 \mathrm{~b}, c$ : dilation of blood vessels $(V)$ and negative staining of neurons. Note pale staining and vacuolation of neuropil tissue (*). 9 d: showing widely separated bundles of the axon (*) and numerous neuroglial cell nuclei ( $\uparrow)$. Orcein stain $a, b$ X200 - c, dX400. 
Orcein stained sections of the cerebellar cortex of hypercholesterolemic rats showed pale staining of the background and loss of purkinje cells and granule cells (Figure $10 \mathrm{a}, \mathrm{b}$ ).

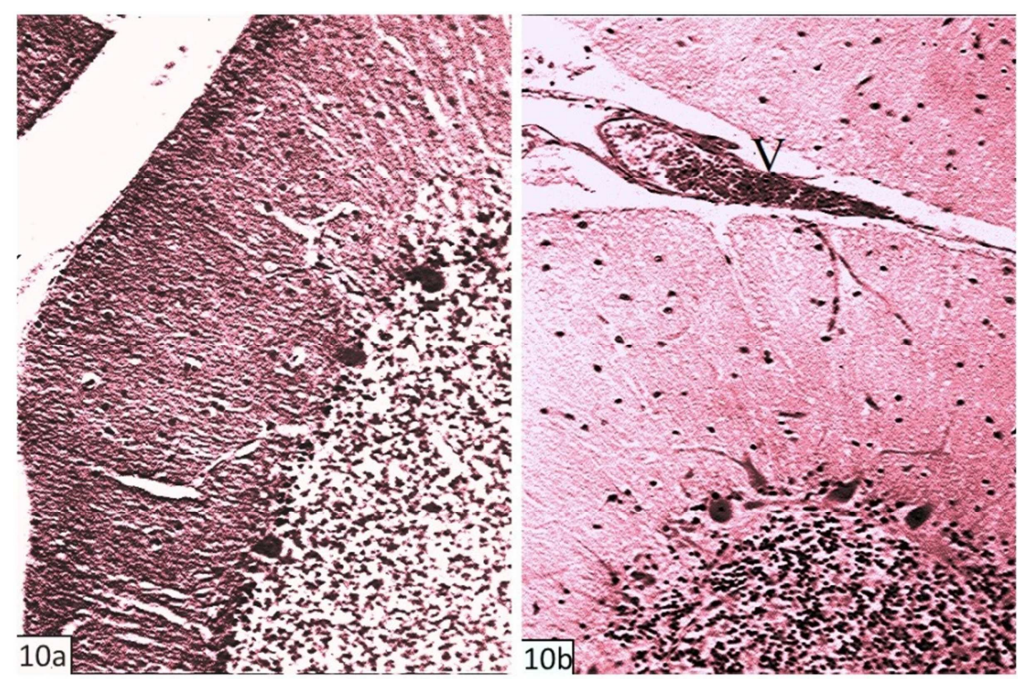

Figure $10 \boldsymbol{a}, \boldsymbol{b}$. Photomicrographs of orcein stained cerebellar cortex of hypercholesterolemic rats showing evident decrease in number of neurons in the three layers of cerebellar cortex associated with congested blood vessels (V). Orcein stain a X100 - b X200.

Orcein stained sections of the frontal cortex of the protected group revealed more or less normal staining pattern as compared with the control group, except mild dilation of the blood vessels (Figure $11 \mathrm{a}, \mathrm{b}$ ). Orcein stained sections of cerebellar cortex of the protected group revealed mild loss of purkinje cells and granule cells (Figure $11 \mathrm{c}$ ).
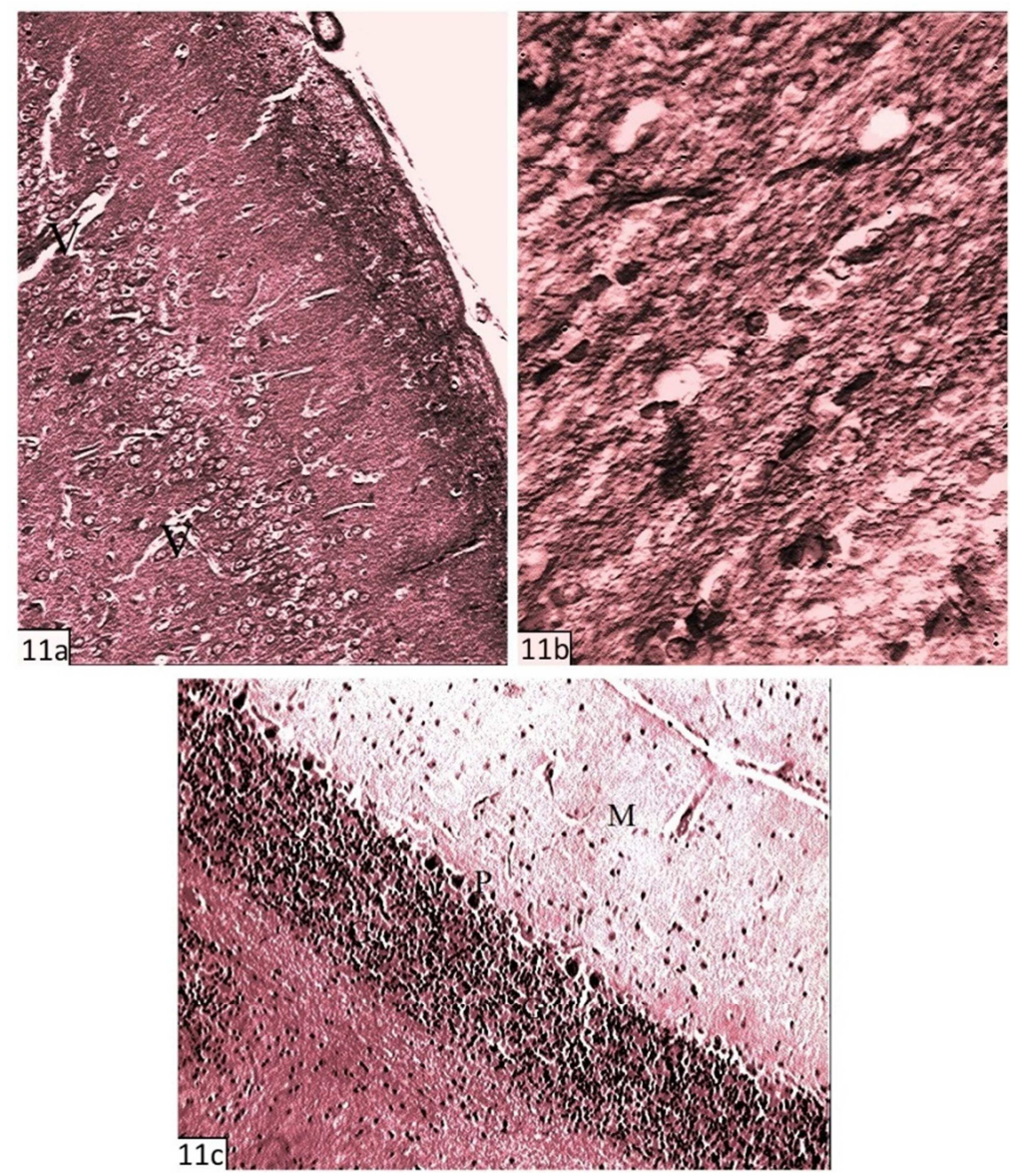

Figure $11 \boldsymbol{a}, \boldsymbol{b}, \boldsymbol{c}$. Photomicrographs of orcein stained frontal and cerebellar cortex of the protected group (received purslane seeds concomitant with high cholesterol diet) showing $11 a$, b: more or less normal orcein staining pattern except mild dilation of the blood vessels (V). 11 c: showing normal architecture of cerebellar cortex as compared with the control group. Orcein stain a, c X200 - b X400. 


\section{Discussion}

Cholesterol is an essential component of cell membranes, which play an important role in the maintenance of cellular homeostasis and transmembrane communication within, and between cellular compartments. Cholesterol is a well-known key regulator of membrane lipid organization and fluidity, and thus homeostatic mechanisms to maintain cellular cholesterol levels in membranes are essential for life. Even though the central nervous system (CNS) represents only $2.2 \%$ of the body weight, it contains as much as $25 \%$ of total body cholesterol. $^{26-28}$

The current study was performed in male rats to exclude hormonal influences, because cholesterol homeostasis of females is mediated by the hypolipidemic properties of estrogens. $^{29}$

In the present study cholesterol-enriched diet significantly increased TG, total cholesterol and LDL- C compared to the control group. However, it is not clear how cholesterol is able to affect brain functions, since cholesterol does not pass the BBB. However oxysterols and oxygenated derivatives of cholesterol are able to pass lipophilic membranes. ${ }^{30-32}$

Consumption of purslane seeds by hypercholesterolemic rats resulted in a significant decrease in lipid parameters as compared with hypercholesterolemic group. Our results suggest that purslane seeds have anti-atherogenic hypolipidemic effects which are probably mediated by unsaturated fatty acids (omega-3) present in the seeds and also the possible influence of the relatively higher fiber content of the seed. Our results are in agreement with Soltan SSA. ${ }^{33}$ The obtained results could be attributed also to the reported antioxidant effects of purslane seeds which in turn lead to decreased free radical generation and decreased oxidative damage of the liver, the main organ involved in cholesterol biosynthesis. ${ }^{18,34}$ It is well known that diet plays an important role in the control of cholesterol homeostasis. It is important to have a balance of omega 3 and omega 6 in the diet. The present study also revealed an increase in HDL-C, which might be due to stimulation of pre- $\beta$ HDL-C and reverse cholesterol transport. High HDL-C levels could potentially contribute to its anti-atherogenic properties, including its capacity to inhibit LDL oxidation and protect endothelial cells from the cytotoxic effects of oxidized LDL.

Histological examination of the grey matter of the frontal cortex of hypercholesterolemic rats revealed various degrees of degenerative changes as evident by distortion of layers, loss of neurons, pyknotic nuclei of degenerated cells and vacuolation of the neuropil associated with proliferation of neuroglial cells. The white matter revealed widely separated bundles of axons. The cerebellum of hypercholesterolemic rats revealed pyknotic nuclei of degenerated cells and loss of purkinje and granule cells.

Cholesterol is an essential molecule for brain homeostasis; the synthesis of cholesterol in the brain occurs mainly in oligodenderocytes and astrocytes. ${ }^{28}$ While it is well known that oligodendrocytes generate cholesterol for myelination process, it has been shown that astrocytes produce cholesterol for neuronal cells. ${ }^{35,36}$ Active axonal growth as well as synapse formation and remodeling requires cholesterol that cannot be provided by distant cell body of the neuron. Cholesterol for these processes may be derived primarily from astrocytes. ${ }^{37}$ Astrocytes are the major glial cell population within the CNS. They play important roles in CNS homeostasis through the release of several neurotrophic factors. This function is crucial for the integrity of the neuronal cytoskeletal networks and the neuronal processes in forming connections with other cells. Brain aging, overt any neurodegenerative state, leads to inflammation, oxidative stress and cell death. Neurons are more susceptible to injury than astrocytes, as they have fewer antioxidant mechanisms and are therefore prone to excitotoxicity. ${ }^{38}$ Both normally and with aging, astrocytes support neurons by providing antioxidant protection, substrates for neuronal metabolism via neurovascular coupling, and glutamate clearance. Although astrocytes are generally more resilient than neurons, severe damage also results in astrocyte dysfunction, leading to increased neuronal death. ${ }^{39}$ Therefore, many recent efforts have focused on the astrocyte-neuron interaction and how astrocyte function can be improved to enhance neuronal support and survival. ${ }^{38}$ A growing body of data demonstrates that astrocytes play a multifaceted and complex role in the response to neuropathologies, including neurodegenerative diseases, as they have potential to both enhance neuronal survival and regeneration and contribute to further injury. ${ }^{40,41}$

Since cholesterol has structural features that make the membranes more rigid, maintaining the proper level of cholesterol is essential for neuronal membrane function, especially at the synaptic ending where membrane fluidity influences the process of neurotransmission. ${ }^{42}$ Cholesterol resident within the brain exists in two pools. $70 \%$ of the total brain cholesterol is metabolically stable and is found in the myelin membranes of white matter ${ }^{43}$ and $30 \%$ is found in the plasma and subcellular membranes of neurons and glial cells within the grey matter. ${ }^{44}$ Almost all of the cholesterol in the brain is synthesized in the brain and little or none of the peripheral cholesterol crosses the BBB. Thus it has been unclear to what extent dietary cholesterol can produce increases in brain cholesterol, ${ }^{45}$ yet hypercholesterolemia and its numerous complications are believed to play a role in promoting multiple aspects of brain pathogenesis. ${ }^{46}$ Clinical observations strongly suggested the presence of exchange between brain and plasma compartments. On the other hand, circulating cholesterol is bound up in LDL and HDL, which enter cells by transcytosis at LDL receptors. There are some controversies regarding the effect of a high cholesterol diet on the central nervous system. It is associated with a lower risk of Parkinson's disease. ${ }^{11,34}$ Microvessels and BBB disruptions induced by elevated cholesterol both in vivo and in vitro which was associated with an increased risk of $\mathrm{AD}$ pathogenesis. There are several proposed mechanisms of hypercholesterolemia induced neurotoxicity. It has been shown that hypercholesterolemia can result in the damage to 
endothelial cells of arteries and capillaries, decrease in blood flow, impairment of metabolism, and the decrease in nutritive and oxygen levels in the brain, thus increasing the possibility of cognitive impairment. It has been reported that hypercholesterolemia impairs endothelial function in brain microvessels and increase the risk of brain infarction. ${ }^{11}$ These findings are in agreement with our results which revealed numerous neuroglial cells, and dilation of the blood vessels.

A great body of evidence has demonstrated that high fat diet has tight relations with vascular damage and oxidative stress so it is possible that hypercholesterolemia facilitates the development of the neurodegenerative disease through increased oxidant production. Several studies have demonstrated that oxidative stress plays an important role in the initiation and progression of atherosclerosis through stimulating inflammation and cytokines production. Inflammation is a common phenomenon in many neurodegenerative diseases. ${ }^{47}$ In $\mathrm{AD}$ and $\mathrm{VaD}$, increased levels of inflammatory proteins in the brain and plasma have been observed even before a clinical onset of dementia. Several studies suggest that inflammation may play a role in BBB dysfunction and cognitive decline. ${ }^{48}$ Brain capillary endothelial cells form the structural basis of the BBB, where they restrict the passage of blood-borne proteins, many neurotoxic agents, and hydrophilic molecules while allowing diffusion of small hydrophobic molecules $\left(\mathrm{O}_{2}\right.$ and $\left.\mathrm{CO}_{2}\right)$ and selective transcellular transport of nutrients and other essential components into the brain parenchyma. ${ }^{49,50}$ The BBB is not a static barrier, but is tightly regulated, allowing changes in vascular permeability. In health, there may be a regulated temporary increase in permeability to allow increased access of nutrients and oxygen to tissues and drug accessibility. However, in disease, an uncontrolled and prolonged increase in BBB permeability results in vasogenic edema and leakage of potentially neurotoxic plasma constituents into tissue. Many acute and chronic neurodegenerative diseases including stroke, multiple sclerosis, rheumatoid arthritis, and acquired immunodeficiency syndrome (AIDS) dementia show changes in BBB integrity. ${ }^{51,52}$ Activated astrocytes and microglia have been shown to increase production of pro-inflammatory cytokines. In fact, preliminary studies have shown increased expression of the pro-inflammatory cytokine tumor necrosis factor- $\alpha$., pharmacological modulation of astrogliosis and microglia activation regulating the $\mathrm{BBB}$ integrity and modification of tight-junction proteins and assess the resulting effect on BBB integrity. ${ }^{53}$

A chronic long-lasting, mild cerebrovascular damage, including inflammatory processes and oxidative stress, may cause AD. Recent studies have shown that high cholesterol levels are linked to the pathology of this disease. These findings are in agreement with the findings in the present study, which demonstrated loss of neurons and pyknotic nuclei of degenerated cells associated with congested dilated blood vessels.

Administration of purslane seeds revealed amelioration evident of most of changes present in frontal cortex and cerebellum. The neuronal activities of purslane were investigated in adult rats by Ahmida et al. ${ }^{54}$ Purslane was able to induce a significant decrease in calcium concentration of the brain cortex. This study concluded that the potential role of purslane for neurotransmitters which is an integral part of many neurodegenerative disorders. Moneim et $\mathrm{al}^{55}$ and $\mathrm{Wang}^{56}$ had reported the neuroprotective activity of oral administration of purslane extracts. The results concluded that the degrees of brain inflammation were reduced due to administration of purslane extracts due to antibacterial, antitumor, anti-inflammatory, vasodilator and immunomodulatory effects. Furthermore, purslane is a rich source of ascorbic acid (AA) which is a famous effective antioxidant. AA is the most predominant form of vitamin $\mathrm{C}$ in the human body and is involved in tissue growth and repair. It is a water-soluble enzyme cofactor, abundantly present in different plants and animals. AA has a powerful antioxidant activity, which made it well known to protect tissues from oxidative injury via efficiently quenching the damaging free radicals produced by different biological processes. ${ }^{54,57-60}$ The antioxidant effect of purslane also mediated through omega-3 content.

\section{Conclusion}

After viewing our results, we found that hyperlipidemia is a highly prevalent risk factor for brain injury. Consequently, hyperlipidemia atherosclerotic lesions were developed in the cerebrovascular system, which led to brain hypoperfusion, oxidative stress and inflammation. The oxidized lipids, especially oxysterol initiated endothelial dysfunction and disruption of the BBB. The damage of the BBB and the existing cerebral ischemia induced neurodegenerative processes in the brain. Various antioxidant strategies are currently being a valuable therapeutic target in many disorders. Therefore, purslane seeds might serve as an adjuvant therapy to avoid progression of brain damage through scavenging of free radicals leading to improvement in brain injury induced by hyperlipidemia.

\section{Conflict of Interest}

None declared

\section{References}

[1] Kruger J, Bowles HR, Jones DA, Ainsworth BE, Kohl HW. Health-related quality of life, BMI and physical activity among US adults (18 years): national physical survey and weight loss survey. Int J Obes. 2002;31:321-327.

[2] Wolf G. After all those years: renal consequences of obesity. Nephrol Dial Transpl. 2003;18:2471-2474.

[3] Xia D, Wu X, Yang Q, Gong J, Zhang Y. Anti-obesity and hypolipidemic effects of a functional formula containing Prumus mume in mice fed high-fat diet. Afr J Biotechnol. 2010;9:2463-2467.

[4] Bell RD, Zlokovic BV. Neurovascular mechanisms and bloodbrain barrier disorder in Alzheimer's disease. Acta Neuropathol. 2009;118:103-113. 
58 El Sayed Aly Mohamed Metwally and Fardous Soror Karawya: Neuroprotective Effects of Purslane Seeds against Adverse Effects Induced by Experimental Hyperlipidemia on Frontal Cortex and Cerebellum in Young Male Albino Rats

[5] Deane R, Zlokovic BV. Role of the blood-brain barrier in the pathogenesis of Alzheimer's disease. Curr Alzheimer Res. 2007;4:191-197

[6] Humpel C, Weis C. Nerve growth factor and cholinergic CNS neurons studied in organotypic brain slices: Implication in Alzheimer's disease? J Neural Transm Suppl. 2002;62:253263.

[7] Heneka MT and O'Banion MK. Inflammatory processes in Alzheimer's disease. J Neuroimmunol. 2007;184: 69-91.

[8] Thirumangalakudi L, Prakasam A, Zhang R, et al. High cholesterol-induced neuroinflammation and amyloid precursor protein processing correlate with loss of working memory in mice. J Neurochem. 2008;106: 475-485.

[9] Ullrich C, Humpel C. Effects of cholesterol and its $24 \mathrm{~S}-\mathrm{OH}$ and 25- $\mathrm{OH}$ oxysterols on choline acetyltransferase - positive neurons in brain slices. Pharmacology. 2010;86:15-21.

[10] Ullrich C, Pirchl M, Humpel C. Hypercholesterolemia in rats impairs the cholinergic system and leads to memory deficits. Mol Cell Neurosci. 2010;45:408-417.

[11] De Lau LM, Koudstaal PJ, Hofman A, Breteler MM. Serum cholesterol levels and the risk of Parkinson's disease. Am J Epidemiol. 2006;164:998-1002.

[12] Morris MC, Evans DA, Bienias JL, et al. Dietary fats and the risk of incident Alzheimer disease. Arch Neurol. 2003; 60:194 200.

[13] Kumar S, Alagawadi KR, Rao MR. Effect of Argyreia speciosa root extract on cafeteria diet-induced obesity in rats. Ind $\mathrm{J}$ Pharmacol. 2011;43:163.

[14] Ellrichmann M, Kapelle M, Ritter PR, et al. Orlistat inhibition of intestinal lipase accurately increase appetite and attenuate post prandial glucagons - like peptide - 7 (7-36)- amide-1 cholesytokinin and peptide yy concentrations. J Clin Endocrinol Metabol. 2008;93:3995-3998.

[15] Radhakrishnan R, Zakaria MN, Islam MW, et al. Neuropharmacological actions of Portulaca oleraceae L V. sativa (Hawk). J Ethnoph armacol. 2001;76:171-176

[16] Rashed AN, Afifi FU, Disi AM. Simple evaluation of the wound healing activity of a crude extract of Portulaca oleracea L. (growing in Jordan) in Mus musculus JVI-1. J Ethnopharmacol. 2003;88: 131-136.

[17] Oiu L, Howe P, Zhou Ye, Xuz, Hocart C, Zhang R. Fatty acids and $\beta$-carotene in Australian purslane (Portulaca oleracea) varieties. J Chromatogr A. 2000; 893:207-213.

[18] Dkhil M, Abdel Moniem A, Al-Quraishy S, Saleh R. Antioxidant effect of purslane and its mechanism of action. J Med Plant Research. 2011;5:1589- 1563.

[19] Al-Quraishy SA, Mohamed A, Dkhil AB, Ahmed E, Abdel Moneim BC. Protective effects of Portulaca oleracea against rotenone mediated depletion of glutathione in the striatum of rats as an animal model of Parkinson's disease. Pesticide Biochemistry and Physiology. 2012;103:108-114.

[20] El-Sayed MI. Effects of Portulaca oleracea L. seeds in treatment of type-2 diabetes mellitus patients as adjunctive and alternative therapy. J Ethnopharmacol. 2011;137:643-651.

[21] Abdalla HM Jr. Purslane extract effects on obesity-induced diabetic rats fed a high-fat diet. Malays J Nutr.
$2010 ; 16: 419-429$.

[22] Rajyalakshmi G, Reddy A, Rajesham VV. A comparative antihyperlipidemic activity of atorvastatin with simvastatin in rats. The Internet Journal of Pharmacology. 2009;6:2.

[23] Friedewald WT, Levy RI, Fredrickson DS. Estimation of the concentration of low-density lipoprotein cholesterol in plasma, without use of the preparative ultracentrifuge. Clin Chem. 1972;18:499-502.

[24] Carletons HM, Drury RAB, Willington EA, Cameron R. Carleton's histological techniques. 5th ed. Oxford: Oxford University press; $1980: 140-222$.

[25] Bancroft, JD, Stevens A. Theory and practice of histological techniques. 2nd ed. Churchill: Livingestone;1982:374-375.

[26] Liu JP, Tang Y, Zhou S, Toh BH, McLean C, Li H. Cholesterol involvement in the pathogenesis of neurodegenerative diseases. Mol Cell Neurosci. 2010;43:33-42.

[27] Dietschy JM, Turley SD. Cholesterol metabolism in the central nervous system during early development and in the mature animal. J Lipid Res. 2004;45: 8:1375-1397.

[28] Pfrieger FW. Cholesterol homeostasis and function in neurons of the central nervous system. Cell Mol Life Sci. 2003;60:1158-1171.

[29] Marinis De, Martini C, Trentalance A, Pallottini V. Sex differences in hepatic regulation of cholesterol homeostasis. J Endocrinol. 2008;198:635-643.

[30] Björkhem I, Lütjohann D, Diczfalusy U, Ståhle L, Ahlborg G, Wahren J. Cholesterol homeostasis in human brain: turnover of 24S-hydroxycholesterol and evidence for a cerebral origin of most of this oxysterol in the circulation. J Lipid Res. 1998;39:1594-1600.

[31] Björkhem I. Crossing the barrier: oxysterols as cholesterol transporters and metabolic modulators in the brain. J Intern Med. 2006;260:493-508.

[32] Vejux A, Malvitte L, Lizard G. Side effects of oxyysterol: cytotoxicity, oxidation, inflammation and phospholipidosis. Braz J Med Biol Res. 2008;41:545-556.

[33] Soltan SSAM. The Effects of varieties sources of omega-3 fatty acids on diabetes in rats. Food and Nutrition Sciences. 2012;3:1404-1412.

[34] Chen Y, Shen Z, Chen X. Evaluation of free radicals scavenging and immunity modulatory activities of purslane polysaccharides. J food Composit Anal. 2007;22:303-306.

[35] Hayashi H, Campenot RB, Vance DE, Vance JE. Glial lipoproteins stimulate axon growth of central nervous system neurons in compartmented cultures. J Biol Chem. 2004;279:1409-1415.

[36] Wahrie SE, Jiang H, Parsadanian $\mathrm{M}$, et al. ABCA1 is required for normal central nervous system apoE levels and for lipidation of astrocyte-secreted apoE. J Biol Chem. 2004;279:40987-40993.

[37] Hering H, Lin CC, Sheng M. Lipid rafts in the maintenance of synapses, dendritic spines, and surface AMPA receptor stability. J Neurosci. 2003;23:3262-3271.

[38] Swanson RA, Ying W, Kauppinen TM. Astrocyte influences on ischemic neuronal death. Curr Mol Med. 2004;4:193-205. 
[39] Nedergaard M, Dirnagl U. Role of glial cells in cerebral ischemia. Glia. 2005;50:281-286.

[40] Sofroniew MV, Vinters HV. Astrocytes: biology and pathology. Acta Neuropathol. 2010;119:7-35.

[41] Sofroniew MV. Molecular dissection of reactive astrogliosis and glial scar formation. Trends Neurosci. 2009;32:638-647.

[42] Simons K, Ehehalt R. Cholesterol, lipid rafts, and disease. J Clin Invest. 2002;110:597-603.

[43] Cuzner ML, Davison AN, Gregson NA. Turnover of brain mitochondrial membrane lipids. Biochem J. 1966;101:618626.

[44] Russell DW, Halford RW, Ramirez DM, Shah R, Kotti T. Cholesterol 24-hydroxylase: an enzyme of cholesterol turnover in the brain. Annu Rev Biochem. 2009;78: 1017-1040

[45] Kirsch C, Eckert GP, Mueller WE. Statin effects on cholesterol micro-domains in brain plasma membranes. Biochem Pharmacol. 2003; 65:843-856.

[46] Hohsfield LA, Daschil N, Orädd G, Strömberg I, Humpel C. Vascular pathology of 20-month-old hypercholesterolemia mice in comparison to triple-transgenic and APPSwDI Alzheimer's disease mouse models. Mol Cell Neurosci. 2014;63:83-95.

[47] Franciosi S, Gama MA, Sosa D, et al. Novel cerebrovascular pathology in mice fed a high cholesterol diet. Mol Neurodegener. 2009;4:42.

[48] Shimizu F, Kanda T. Disruption of blood -brain barrier in inflammatory neurological diseases. Brain Nerve. 2013;65:165-76.

[49] Abbott NJ. Astrocyte-endothelial interactions and blood-brain barrier permeability. J Anat. 2002;200;629-638.

[50] Wolburg H, Lippoldt A. Tight junctions of the blood-brain barrier: Development, composition and regulation. Vasc Pharmacol. 2002;38:323-337.

[51] Plumb J, McQuaid S, Mirakhur M, Kirk J. Abnormal endothelial tight junctions in active lesions and normal-appearing white matter in multiple sclerosis. Brain Pathol. 2002;12:154-169.

[52] Abbott NJ, Ronnback L, Hansson E. Astrocyte-endothelial interactions at the blood-brain barrier. Nat Rev Neurosci. 2006;7:41-53.

[53] Willis CL, Nolan CC, Reith SN, et al. Focal astrocyte loss is followed by microvascular damage, with subsequent repair of the blood-brain barrier in the apparent absence of direct astrocytic contact. Glia. 2004;45:325-337.

[54] Ahmida MH. Evaluation of in vivo antioxidant and hepatoprotective activity of $\mathrm{P}$. oleracea against paracetamol induced liver toxicity in male rats. American Journal of Pharmacology and Toxicology. 2010;5:167-176.

[55] Moneim AEA, Nasr I, Dkhil MA, Quraishy S. Neuronal activities of P. oleracea in adult rats. Journal of Medicinal Plants Research. 2012;6:3162-3168.

[56] Wang CQ, Yang GQ. Betacyanins from Portulaca oleracea L. ameliorate cognition deficits and attenuate oxidative damage induced by D-galactose in the brains of senescent mice. Phytomedicine. 2010;17:527-532.

[57] Karimi G, Khoei A, Omidi A, et al. Protective effect of aqueous and ethanolic extracts of P. oleracea against cisplatin induced nephrotoxicity. Iranian Journal of Basic Medical Sciences. 2010;13:31-35.

[58] Besong SA, Ezekwe MO, Ezekwe EI. Evaluating the effects of freeze-dried supplements of purslane (P. oleracea) on blood lipids in hypercholesterolemic adults. International Journal of Nutrition and Metabolism. 2011; 3:43-49.

[59] Ballard C, Gauthier S, Corbett A, Brayne C, Aarsland D, Jones E. Alzheimer's disease. Lancet. 2011;377:1019-1031.

[60] Xue QS, Sparks DL, Streit WJ. Microglial activation in the hippocampus of hypercholesterolemic rabbits occurs independent of increased amyloid production. J Neuroinflammation. 2007;4:20. 